\title{
INTERIOR SPHERE PROPERTY FOR LEVEL SETS OF THE VALUE FUNCTION OF AN EXIT TIME PROBLEM
}

\author{
Marco Castelpietra ${ }^{1}$
}

\begin{abstract}
We consider an optimal control problem for a system of the form $\dot{x}=f(x, u)$, with a running cost $L$. We prove an interior sphere property for the level sets of the corresponding value function $V$. From such a property we obtain a semiconcavity result for $V$, as well as perimeter estimates for the attainable sets of a symmetric control system.
\end{abstract}

Mathematics Subject Classification. 93B03, 49L20, 49L25

Received November 25, 2006. Revised September 18, 2007.

Published online March 6, 2008.

\section{INTRODUCTION}

Fix a closed set $\mathcal{K}$, the target, and consider a control system of the form

$$
\left\{\begin{array}{l}
\dot{y}(t)=f(y(t), u(t)) \quad u(t) \in U \subseteq \mathbb{R}^{m} \\
y(0)=x
\end{array}\right.
$$

and a cost functional $J(t, x, u)=\int_{0}^{t} L\left(y\left(t^{\prime}\right), u\left(t^{\prime}\right)\right) \mathrm{d} t^{\prime}$. The exit time for a trajectory $y^{x, u}(t)$ is $\tau(x, u)=\min \{t \geqslant$ $\left.0: y^{x, u}(t) \in \mathcal{K}\right\}$. We shall be concerned with the optimal control problem, which consists in minimizing, for a given $x \in \mathbb{R}^{n}$, the cost $J(\tau(x, u), x, u)$ over all the controls $u$. The value function of such a problem is

$$
V_{\mathcal{K}}(x)=\inf _{u(\cdot)} J(\tau(x, u), x, u) .
$$

In this paper we are interested to study the regularity of $V_{\mathcal{K}}$ and its level sets. Some standard assumptions allow to recover Lipschitz continuity (see [5]). If we consider a trivial running cost $L \equiv 1$, the value function turns out to be the minimum time function $T_{\mathcal{K}}(x)=\inf _{u(\cdot)} \tau(x, u)$. If the target $\mathcal{K}$ is regular, and if the control system satisfies the Petrov condition, then $T_{\mathcal{K}}$ turns out to be semiconcave (see for instance [4]). This semiconcavity result was also extended to the value function in the case of nonconstant $L$ (see [6]). Cannarsa and Frankowska (in [3]) prove the local semiconcavity of $T_{\mathcal{K}}$ also in the case of non regular target $\mathcal{K}$. They observe that the level sets of the minimum time function are related with the attainable sets from $\mathcal{K}$ in time $t$, i.e. $\mathcal{A}(\mathcal{K}, t)=\left\{y^{x, u}(t): x \in \mathcal{K}, u(\cdot)\right.$ control $\}$, for a suitable choice of $f$. They prove the Interior Sphere Property

\footnotetext{
Keywords and phrases. Control theory, interior sphere property, value function, semiconcavity, perimeter.

1 Dipartimento di Matematica, Università di Roma "Tor Vergata", Via della Ricerca Scientifica 1, 00133 Roma, Italy.

castelpi@mat.uniroma2.it
} 
of these sets, and they apply this property to the level sets of $T_{\mathcal{K}}$. So they obtain a local semiconcavity result, using a Petrov type condition. Still considering non regular target $\mathcal{K}$, Sinestrari (in [8]) proves local semiconcavity of $V_{\mathcal{K}}$ for nonconstant $L$. The proof uses a direct approach to the problem, and does not involve the analysis of regularity properties of the level sets.

We study the attainable sets, in the problem with a non trivial running cost. So, for general closed target $\mathcal{K}$, we recover an Interior Sphere Property for the level sets of $V_{\mathcal{K}}$, and, under Petrov's condition, local semiconcavity follows. To obtain regularity results for the level sets of $V_{\mathcal{K}}$, we turn our attention to a suitable time optimal problem, for which the minimum time function turns out to be equal to the value function $V_{\mathcal{K}}$ of the original problem. We use a natural correspondence between the trajectories of system (1.1) and the trajectories of the system with dynamics $\bar{f}(x, u)=f(x, u) / L(x, u)$. The above correspondence is obtained by a rescaling of the time, and preserves the images of the arcs. So a discussion of regularity of $L$ allows to obtain the Interior Sphere Property for the level sets of $V_{\mathcal{K}}$. Moreover we show an application of the Interior Sphere Property to the analysis of the perimeters of the level sets of the value function. For the attainable sets in time $t$ the problem was studied by Alvarez, Cardaliaguet and Monneau (in [1]), and by Cannarsa and Cardaliaguet (in [2]). We show that, for a system of the form $\dot{y}=f(y) u$, the above results can be extended to the case of a nonconstant $L$.

This paper is structured as follows. Section 2 is devoted to notations, definitions, basic results and assumptions. In Section 3, we study the equivalence between a control systems with running cost $L$ and a control system with a trivial running cost. In Section 4 we analyse the assumptions that we need to obtain the Interior Sphere Property (using the above equivalence result). Finally, in Sections 5 and 6 we apply these results to the semiconcavity of the value function $V$ and to the growth estimates for perimeters.

\section{Preliminaries}

We denote by $\langle\cdot, \cdot\rangle$ the Euclidean scalar product in $\mathbb{R}^{n}$, and by $|\cdot|$ its norm. For any $x \in \mathbb{R}^{n}$ and any $r>0$, we set $B(x, r)=\left\{y \in \mathbb{R}^{n}:|y-x|<r\right\}$, or equivalently $B_{r}(x)$. We also use the abbreviations $B(r)=B r=B(0, r)$ and $B=B(1)$ (so $x+r B=B_{r}(x)$ ).

For any subset $S \subseteq \mathbb{R}^{n}$, we denote by $\bar{S}$ its closure, $\partial S$ the boundary, and $S^{c}=\mathbb{R}^{n} \backslash S$ the complement.

If $S$ is a measurable set, we indicate with $|S|$ its Lebesgue measure in $\mathbb{R}^{n}$. And we denote by $\mathcal{H}^{k}(S)$ the Hausdorff measure of dimension $k$. For details on the Hausdorff measure, see for instance [7].

We denote by $d_{S}$ the distance function from $S$, defined as

$$
d_{S}(x)=\inf _{y \in S}|x-y| \quad x \in \mathbb{R}^{n}
$$

and by $b_{S}$ the signed distance from $S$, that is

$$
b_{S}(x)=d_{S}(x)-d_{S^{c}}(x) .
$$

The contingent cone to $S$ at a point $x \in S$ is

$$
T_{S}(x)=\left\{v \in \mathbb{R}^{n}: \liminf _{v \searrow 0} \frac{d_{S}(x+t v)}{t}=0\right\} .
$$

The normal cone to $S$ at a point $x \in S$ is

$$
N_{S}(x)=\left\{p \in \mathbb{R}^{n}:\langle p, v\rangle \leqslant 0, \forall v \in T_{S}(x)\right\}
$$

The projection of $x$ onto $S$ is the set

$$
\pi_{S}(x)=\left\{y \in \bar{S}:|x-y|=d_{S}(x)\right\}
$$


For simplicity, when the projection is unique, we shall identify a point $y$ with the singleton $\{y\}$. Hence $\pi_{S}(x)=y$ or $\pi_{S}(x)=\{y\}$ will mean the same.

Let us recall the definition of Interior Sphere Property and semiconcavity, which are the regularity properties we will investigate in the following sections.

Definition 2.1. Let $S$ be closed and $r>0$. We say that $S$ has the Interior Sphere Property (or ISP) of radius $r$ at a point $x \in \partial S$ if $x$ belongs to some closed ball $y_{x}+r \bar{B} \subseteq S$. We say that $S$ has the (uniform) Interior Sphere Property of radius $r$ (or r-ISP) if $S$ has the ISP of radius $r$ at every point $x \in \partial S$.

Definition 2.2. A continuous function $\varphi: \Omega \rightarrow \mathbb{R}$, with $\Omega \subseteq \mathbb{R}^{n}$, is called semiconcave if there exists $C>0$ such that

$$
\varphi(x+h)+\varphi(x-h)-2 \varphi(x) \leqslant C|h|^{2},
$$

for all $x \in \Omega$, and for all $h \in \mathbb{R}^{n}$ such that $[x-h, x+h] \subseteq \Omega$. The constant $C$ is called a semiconcavity constant for $\varphi$ in $\Omega$. We call $\operatorname{SC}_{l o c}(\Omega)$ the class of the functions which are semiconcave on all compact subsets of $\Omega$.

Remark 2.3. A function $\varphi$ is semiconcave if and only if the function $x \mapsto \varphi(x)-C|x|^{2}$ is concave. Any semiconcave function in $\Omega$ is also locally Lipschitz continuous in $\Omega$, since so are concave functions.

For more details on Interior Sphere Property and semiconcavity (and their links) we refer the reader to [5]. Now let us describe the system we will study, with the assumption under which we consider it.

\subsection{Description of the system and basic assumptions}

Let a compact set $U \subset \mathbb{R}^{m}, m \geqslant 1$, and a map $f: \mathbb{R}^{n} \times U \rightarrow \mathbb{R}^{n}$ be given. We define the set

$$
F(x):=f(x, U)
$$

of the admissible velocities at a point $x \in \mathbb{R}^{n}$. For any point $x \in \mathbb{R}^{n}, T>0$, and any measurable function $u:[0, T] \rightarrow U$, we consider the equation

$$
\left\{\begin{array}{l}
\dot{y}(t)=f(y(t), u(t)), \quad \text { a.e. } t \geqslant 0 \\
y(0)=x
\end{array}\right.
$$

The measurable function $u(t)$ is called an admissible control. Assume that

$f$ is continuous and $\exists L_{0}>0$ such that $f(\cdot, u)$ is Lipschitz continuous on $\mathbb{R}^{n}$ of rank $L_{0}$ for every $u \in U$.

Then, for any fixed $x$ and $u(\cdot)$, the Cauchy problem $(2.1)$ has a unique solution in $[0, T]$, that we call

$$
y(t ; x, u)=y^{x, u}(t)
$$

We are interested in studying the set of the points that we can reach by a trajectory of $(2.1)$. Let $\mathcal{K} \subseteq \mathbb{R}^{n}$ be a closed set.

Definition 2.4. For any $t \geqslant 0$, the attainable set from $\mathcal{K}$ at time $t$ is

$$
\mathcal{A}(\mathcal{K}, t)=\left\{y(t ; x, u): x \in \mathcal{K}, u \in \mathrm{L}^{1}([0, t], U)\right\}
$$

If $F(x)$ is convex for every $x \in \mathbb{R}^{n}$ then the set $\mathcal{A}(\mathcal{K}, t)$ is closed for any $t \geqslant 0$. 
Remark 2.5. Owing to assumption (2.2), for any $x \in \mathbb{R}^{n}$ and for any $T>0$ there exists $R_{x, T}>0$ such that

$$
y(t ; x, u) \in x+R_{x, T} \bar{B} \quad \forall t \in[0, T]
$$

for any admissible control $u$. The application $(x, T) \mapsto R_{x, T}$ can be supposed to be continuous. Vice versa, for any $z \in \mathbb{R}^{n}$ and for any $T>0$ there exists $\hat{R}_{z, T}$ such that, if $z=y(t ; x, u)$ with $t \in[0, T]$, then $x \in z+\hat{R}_{z, T} \bar{B}$. Moreover, if $\sup _{\mathcal{K} \times U}|f(x, u)|<+\infty$, then for any $T>0$ there exists $R_{T}>0$ such that

$$
\mathcal{A}(\mathcal{K}, t) \subseteq \mathcal{K}+R_{T} \bar{B} \quad \forall t \in[0, T]
$$

Let $L: \mathbb{R}^{n} \times U \rightarrow \mathbb{R}$ (running cost) be a continuous function and let $J$ be the cost functional

$$
J(t, x, u)=\int_{0}^{t} L\left(y^{x, u}\left(t^{\prime}\right), u\left(t^{\prime}\right)\right) \mathrm{d} t^{\prime} .
$$

On the running cost $L$ we assume that there exists a number $k_{0} \in \mathbb{R}$ such that

$$
L(x, u) \geqslant k_{0}>0 \quad \forall x \in \mathbb{R}^{n}, \forall u \in U
$$

In the problem with nonconstant $L$, we also define the set that is the generalization of the attainable set in time $t$.

Definition 2.6. Let $\mathcal{K} \subseteq \mathbb{R}^{n}$ be a closed set, and $\lambda \geqslant 0$. The attainable set from $\mathcal{K}$ with cost $\lambda$ is

$$
\mathcal{A}_{L}(\mathcal{K}, \lambda)=\left\{y^{x, u}(t): J(t, x, u)=\lambda, \text { for some } t \geqslant 0, x \in \mathcal{K}, u \in \mathrm{L}^{1}([0, t], U)\right\}
$$

The attainable set in time $t, \mathcal{A}(\mathcal{K}, t)$, depends only on $f$ (and $\mathcal{K}$ ); whereas the attainable set with cost $\lambda$, $\mathcal{A}_{L}(\mathcal{K}, \lambda)$, depends also on the running cost $L$. We are interested in these two sets because $\mathcal{A}(\mathcal{K}, t)$ and $\mathcal{A}_{L}(\mathcal{K}, \lambda)$ are related with the level sets of, respectively, the minimum time function $T_{\mathcal{K}}$ and the value function $V_{\mathcal{K}}$. In Section 5 we will give more details about this relation.

The properties of the set $\mathcal{A}(\mathcal{K}, t)$ are well-known (see for instance [3]), therefore we are interested in the regularity properties of the set $\mathcal{A}_{L}(\mathcal{K}, \lambda)$. So, we will assume:

$$
\left\{\begin{aligned}
\text { (i) } & F(x) \text { is convex for every } x \in \mathbb{R}^{n} ; \\
\text { (ii) } & \text { for any compact set } \mathcal{X} \subset \mathbb{R}^{n}, \text { there exists a number } \\
& r_{\mathcal{X}}>0 \text { such that } F(x) \text { has the Interior Sphere Property } \\
& \text { of radius } r_{\mathcal{X}} \text { for every } x \in \mathcal{X} ; \\
\text { (iii) } & f(\cdot, u) \text { is differentiable for every } u \in U, \text { and for any } \\
& \text { compact set } \mathcal{X} \subset \mathbb{R}^{n}, \text { there exists } L_{1}(\mathcal{X})>0 \text { such that, } \\
& \text { for any } x, y \in \mathcal{X} \\
& \left\|f_{x}(x, u)-f_{x}(y, u)\right\| \leqslant L_{1}(\mathcal{X})|x-y| \quad \forall u \in U
\end{aligned}\right.
$$

where $f_{x}$ denotes the Jacobian matrix of $f(x, u)$ with respect to $x$. We say that assumption (2.4) holds globally if $r \equiv r_{\mathcal{X}}$ and $L_{1} \equiv L_{1}(\mathcal{X})$ are independent of the compact set $\mathcal{X}$.

\subsection{Preliminary results}

In view of our assumptions, we have some immediate consequence. In the following proposition, the Lipschitz regularity of $f$ yields an estimate for the distance between the sets of admissible velocities $F(x)$; from the Interior Sphere Property of the sets $F(x)$, we can deduce a Lipschitz constant for $\nabla b_{F(x)}$. 
Proposition 2.7. Assume that (2.2), (2.4)(i) and (2.4)(ii) are satisfied, let $\mathcal{X} \subset \mathbb{R}^{n}$ be a compact set and let $x, y \in \mathcal{X}$. Then

(a) for all $v \in \partial F(x),\left|b_{F(y)}(v)\right| \leqslant L_{0}|x-y|$;

(b) on $\partial F(x)+\frac{r_{\mathcal{X}}}{2} B$, the map $v \mapsto b_{F(x)}(v)$ is $\mathrm{C}^{1,1}$, and

$$
\left|\nabla b_{F(x)}(v)-\nabla b_{F(x)}\left(v^{\prime}\right)\right| \leqslant \frac{2}{r_{\mathcal{X}}}\left|v-v^{\prime}\right| \quad \forall v, v^{\prime} \in \partial F(x)+\frac{r_{\mathcal{X}}}{2} B .
$$

For the proof we refer the reader to Proposition 3.1 of [3]. These results, that are useful to study the regularity of the attainable set in time $t$, can also be helpful when we consider a nontrivial running cost.

Cannarsa and Frankowska, in [3], proved the ISP for the sets $\mathcal{A}(\mathcal{K}, t)$ using assumptions (2.2) and (2.4) and a regularity hypothesis on $F(x)$ described in the following.

Definition 2.8. Let $\mathcal{X} \subseteq \mathbb{R}^{n}$ be a closed set. We say that $x \rightsquigarrow \partial F(x)$ is a Lipschitz boundary map on $\mathcal{X}$ if there exist $r_{0} \in\left(0, \frac{r_{\mathcal{X}}}{2 L_{0}}\right]$ and $C_{0} \geqslant 0$ such that

$$
\left|\nabla b_{F(y)}(v)-\nabla b_{F(x)}(v)\right| \leqslant C_{0}|x-y|
$$

for all $x \in \mathcal{X}$, all $y \in \bar{B}\left(x, r_{0}\right)$, and all $v \in \partial F(x)$. If the property holds true for $\mathcal{X}=\mathbb{R}^{n}$ we say, simply, that $x \rightsquigarrow \partial F(x)$ is a Lipschitz boundary map.

As usual, we will say that $x \rightsquigarrow \partial F(x)$ is a locally Lipschitz boundary map if the property holds on any compact set of $\mathbb{R}^{n}$. In Section 4 we will give a sufficient condition to ensure that (2.5) is satisfied, but for a more exhaustive exposition we refer the reader to [3].

Theorem 2.9 (Th. 3.8 of [3]). Let $\mathcal{K} \subseteq \mathbb{R}^{n}$ be a closed set. Assume that (2.2) and (2.4) hold globally. Suppose that

$$
|f(x, u)| \leqslant H \quad \forall(x, u) \in \mathcal{K} \times U
$$

for some constant $H>0$. If $x \rightsquigarrow \partial F(x)$ is a Lipschitz boundary map, then for any $T_{0}>0$ there exists $\rho>0$ such that, for all $T \in\left(0, T_{0}\right), \mathcal{A}(\mathcal{K}, T)$ has the ISP of radius $\rho T$.

\section{An equivalence Result}

In this section we consider a control system of the form (2.1) and a cost $J(t, x, u)$. We show that this is equivalent to study another control system with running $\operatorname{cost} \bar{L} \equiv 1$, that is $\bar{J}(t, x, u)=t$.

Let us consider the control system

$$
\left\{\begin{array}{l}
\frac{\mathrm{d}}{\mathrm{d} t} y(t)=f(y(t), u(t)), \quad u(\cdot) \text { is an admissible control } \\
y(0)=\zeta \in \mathcal{K} .
\end{array}\right.
$$

We want to investigate the properties of the attainable set $\mathcal{A}_{L}(\mathcal{K}, \lambda)$, where $\lambda$ is the cost $\lambda=J(t, \zeta, u):=$ $\int_{0}^{t} L\left(y^{\zeta, u}\left(t^{\prime}\right), u\left(t^{\prime}\right)\right) \mathrm{d} t^{\prime}$. Define the set of the related control pairs

$$
\mathcal{S}(\zeta):=\left\{\left(y^{\zeta}, u\right): y^{\zeta}(\cdot)=y^{\zeta, u}(\cdot) \text { is solution to }(3.1)\right\}
$$

Now we define another control system, for which we consider the attainable set in time $s$. Recall that $L$ is bounded from below by $k_{0}>0$, and consider the dynamics

$$
\bar{f}(x, u):=\frac{1}{L(x, u)} f(x, u)
$$


with the control system

$$
\left\{\begin{array}{l}
\frac{\mathrm{d}}{\mathrm{d} s} x(s)=\bar{f}(x(s), v(s)), \quad v(\cdot) \text { is an admissible control } \\
x(0)=\zeta \in \mathcal{K} .
\end{array}\right.
$$

For this control system we consider the "cost" $\bar{J}(s, \zeta, v):=s$. We also define the set of control pairs

$$
\overline{\mathcal{S}}(\zeta):=\left\{\left(x^{\zeta}, v\right): x^{\zeta}(\cdot)=x^{\zeta, v}(\cdot) \text { is solution to }(3.2)\right\}
$$

Furthermore, we set

$$
\mathcal{S}(\mathcal{K}):=\bigcup_{\zeta \in \mathcal{K}} \mathcal{S}(\zeta), \quad \overline{\mathcal{S}}(\mathcal{K}):=\bigcup_{\zeta \in \mathcal{K}} \overline{\mathcal{S}}(\zeta)
$$

For simplicity we start analyzing the simplest case $\mathcal{K}=\{0\}$ (then $y(0)=x(0)=0)$. We will use the notations

$$
y^{u}(t):=y^{0, u}(t), \mathcal{A}(t):=\mathcal{A}(\{0\}, t), \mathcal{A}_{L}(t):=\mathcal{A}_{L}(\{0\}, t), J(t, u):=J(t, 0, u),
$$

and $\mathcal{S}:=\mathcal{S}(0)$

We want to show that system (3.1) is "equivalent" to system (3.2). That is, each trajectory of (3.1) is also a trajectory of (3.2) (up to a change of parameter). At this aim we define a one to one correspondence $\varphi: \mathcal{S} \rightarrow \overline{\mathcal{S}}$ as follows. Fix $(y, u) \in \mathcal{S}$. Then $y=y^{u}$ is a solution of $(3.1)$ on some interval $[0, \tau]$. Define the change of parameter $h_{y}:[0, \tau] \rightarrow \mathbb{R}$ as

$$
s=h_{y}(t):=\int_{0}^{t} L\left(y\left(t^{\prime}\right), u\left(t^{\prime}\right)\right) \mathrm{d} t^{\prime} .
$$

As $L$ is positive, $h_{y}(t)$ is strictly increasing, then there exists the inverse function $h_{y}^{-1}(s)$. So the pair of functions

$$
\varphi(y, u):=\left(y \circ h_{y}^{-1}, u \circ h_{y}^{-1}\right)
$$

is defined on the interval $\left[0, h_{y}(\tau)\right]$.

Once $\varphi(y, u)$ is defined, it remains to show if $\varphi(y, u) \in \overline{\mathcal{S}}$ and if the application $\varphi$ is invertible. This is the target of the following proposition.

Proposition 3.1. Let $\mathcal{K}=\{0\}$. Assume (2.2) and (2.3). Then there exists a one to one correspondence $\varphi: \mathcal{S} \rightarrow \overline{\mathcal{S}}$.

Proof. Let $\varphi$ be the application defined by (3.3) and (3.4). We split the proof in three steps.

Step 1. $(y, u) \in \mathcal{S} \Longrightarrow \varphi(y, u) \in \overline{\mathcal{S}}$.

Let $(y, u)$ a control pair of $(3.1)$ in $[0, \tau]$, and let $(x, v):=\varphi(y, u)$. For any $s \in\left[0, h_{y}(\tau)\right]$ we have that $x(s)=y\left(h_{y}^{-1}(s)\right)$, and $v(s)=u\left(h_{y}^{-1}(s)\right)$. This is equivalent to write

$$
x\left(h_{y}(t)\right)=y(t), \quad v\left(h_{y}(t)\right)=u(t)
$$

Since $(y, u) \in \mathcal{S}$, we have that, for any $t \in[0, \tau]$,

$$
\begin{aligned}
f(y(t), u(t)) & =\frac{\mathrm{d}}{\mathrm{d} t} y(t)=\frac{\mathrm{d}}{\mathrm{d} t} x\left(h_{y}(t)\right)=\frac{\mathrm{d}}{\mathrm{d} s} x\left(h_{y}(t)\right) \cdot \frac{\mathrm{d}}{\mathrm{d} t} h_{y}(t) \\
& =\frac{\mathrm{d}}{\mathrm{d} s} x\left(h_{y}(t)\right) \cdot L(y(t), u(t))
\end{aligned}
$$


and then

$$
\begin{aligned}
\frac{\mathrm{d}}{\mathrm{d} s} x\left(h_{y}(t)\right) & =\frac{1}{L(y(t), u(t))} f(y(t), u(t))=\bar{f}(y(t), u(t)) \\
& =\bar{f}\left(x\left(h_{y}(t)\right), v\left(h_{y}(t)\right)\right) .
\end{aligned}
$$

It follows that, for every $s \in\left[0, h_{y}(\tau)\right]$,

$$
\frac{\mathrm{d}}{\mathrm{d} s} x(s)=\bar{f}(x(s), v(s)) .
$$

Moreover, by definition, $x(0)=y\left(h_{y}^{-1}(0)\right)=y(0)$. Therefore $\varphi(y, u) \in \overline{\mathcal{S}}$.

Step 2. Define an application $\psi: \overline{\mathcal{S}} \rightarrow \mathcal{S}$.

We define $\psi$ in analogous way to $\varphi$, but with a different change of parameter. Let $(x, v)$ a control pair of (3.2) in $[0, \sigma]$. Define $g_{x}:[0, \sigma] \rightarrow \mathbb{R}$ as

$$
t=g_{x}(s):=\int_{0}^{s} \frac{1}{L\left(x\left(s^{\prime}\right), v\left(s^{\prime}\right)\right)} \mathrm{d} s^{\prime} .
$$

The definition of $g_{x}$ is well posed, and it is well defined the inverse $g_{x}^{-1}$. If we set

$$
\psi(x, v):=\left(x \circ g_{x}^{-1}, v \circ g_{x}^{-1}\right)
$$

and we argue in the same way of the previous step, we find that $\psi(x, v) \in \mathcal{S}$ for any $(x, v) \in \overline{\mathcal{S}}$ (and $\psi$ is well defined).

Step 3. $\psi=\varphi^{-1}$.

Fix $\left(y_{0}, u_{0}\right) \in \mathcal{S}$ and let $\left(x_{0}, v_{0}\right)=\varphi\left(y_{0}, u_{0}\right)$ via the function $h_{0}$, and $\left(y_{1}, u_{1}\right)=\psi\left(x_{0}, v_{0}\right)$ via the function $g_{0}$.

To conclude this step we have to check that $\left(y_{0}, u_{0}\right)=\left(y_{1}, u_{1}\right)$. We recall that, by hypothesis,

$$
\begin{aligned}
& y_{0}(t)=x_{0}\left(h_{0}(t)\right) \text { and } u_{0}(t)=v_{0}\left(h_{0}(t)\right) \\
& y_{1}(t)=x_{0}\left(g_{0}^{-1}(t)\right) \text { and } u_{1}(t)=v_{0}\left(g_{0}^{-1}(t)\right) .
\end{aligned}
$$

It follows that, if $h_{0}=g_{0}^{-1}$, we conclude the proof. Now, for any $t \geqslant 0$,

$$
\begin{aligned}
t & =\int_{0}^{t} \mathrm{~d} t^{\prime}=\int_{0}^{h_{0}(t)} \frac{\mathrm{d} h_{0}^{-1}\left(s^{\prime}\right)}{\mathrm{d} s^{\prime}} \mathrm{d} s^{\prime}=\int_{0}^{h_{0}(t)} \frac{1}{\frac{\mathrm{d}}{\mathrm{d} t^{\prime}} h_{0}\left(h_{0}^{-1}\left(s^{\prime}\right)\right)} \mathrm{d} s^{\prime} \\
& =\int_{0}^{h_{0}(t)} \frac{1}{L\left(y_{0}\left(h_{0}^{-1}\left(s^{\prime}\right)\right), u_{0}\left(h_{0}^{-1}\left(s^{\prime}\right)\right)\right)} \mathrm{d} s^{\prime}=\int_{0}^{h_{0}(t)} \frac{1}{L\left(x_{0}\left(s^{\prime}\right), v_{0}\left(s^{\prime}\right)\right)} \mathrm{d} s^{\prime} \\
& =g_{0}\left(h_{0}(t)\right),
\end{aligned}
$$

and, by invertibility of $g_{0}$ and $h_{0}$, we have that $h_{0}=g_{0}^{-1}$.

Once we have a one to one correspondence between $\mathcal{S}$ and $\overline{\mathcal{S}}$, it would be useful to see how do $\varphi$ and $\psi$ affect the cost $J$ and $\bar{J}$. The following proposition shows that the cost is an invariant for this correspondence.

Proposition 3.2. Assume (2.2) and (2.3). Let $h_{y}$ and $\varphi$ be the applications defined, respectively, in (3.3) and (3.4). For any $(y, u) \in \mathcal{S}$ and for any $t \geqslant 0$ we have that

$$
J(t, u)=\bar{J}\left(h_{y}(t), \varphi_{2}(y, u)\right),
$$

where $\varphi_{2}(\cdot)$ is the second component of $\varphi(\cdot)$. 
Proof. Consider $(x, v)=\varphi(y, u)$ and call $s=h_{y}(t)$. By definition of $h_{y}$ and $J$, we have that

$$
\bar{J}(s, v)=s=h_{y}(t)=\int_{0}^{t} L(y(\tau), u(\tau)) \mathrm{d} \tau=J(t, u),
$$

and this concludes the proof.

It follows that $\mathcal{A}_{L}(\lambda)=\overline{\mathcal{A}}(\lambda)$, where $\mathcal{A}_{L}(\lambda)$ is the attainable set with cost $\lambda$ for system (3.1), and $\overline{\mathcal{A}}(\lambda)$ is the attainable set at time $\lambda$ for system (3.2). Indeed, from Propositions 3.1 and 3.2, we have that

$$
\begin{aligned}
\mathcal{A}_{L}(\lambda) & =\left\{y^{u}(t): J(t, u)=\lambda, \text { for some } t \geqslant 0, u \text { admissible control }\right\} \\
& =\left\{x^{v}(\lambda): v \text { admissible control }\right\} \\
& =\overline{\mathcal{A}}(\lambda) .
\end{aligned}
$$

It is clear that this equality is independent from the starting point. Hence the result holds true even if we consider the system starting from a closed set $\mathcal{K} \subseteq \mathbb{R}^{n}$.

Corollary 3.3. Let $\mathcal{K} \subseteq \mathbb{R}^{n}$ be a closed set and $\lambda>0$. Assume (2.2) and (2.3). Let $\mathcal{A}_{L}(\mathcal{K}, \lambda)$ be the attainable set with cost $\lambda$ for system (3.1), and $\overline{\mathcal{A}}(\mathcal{K}, \lambda)$ the attainable set in time $\lambda$ for system (3.2). Then

$$
\mathcal{A}_{L}(\mathcal{K}, \lambda)=\overline{\mathcal{A}}(\mathcal{K}, \lambda)
$$

\section{INTERIOR Sphere Property}

In this section we consider control system (2.1) and we show that, under suitable assumptions on $L$, the set $\mathcal{A}_{L}(\mathcal{K}, \lambda)$ has the Interior Sphere Property. In view of Corollary 3.3 we can use some known result for the attainable set in time $t$ for system (3.2). If $\bar{f}(x, u)=\frac{1}{L(x, u)} f(x, u)$ satisfies the assumptions of Theorem 2.9, we obtain an Interior Sphere Property for $\mathcal{A}_{L}(\mathcal{K}, \lambda)$. At this aim we will consider a more restrictive running cost

$$
L: \mathbb{R}^{n} \rightarrow \mathbb{R}
$$

since it is very difficult to obtain the regularity properties needed for $\bar{f}$, even if $L(x, \cdot)$ is very regular.

In the following we give an example of a control system with a running cost $L$ that is very simple (and regular w.r.t. $u$ ) for which $\mathcal{A}_{L}(\mathcal{K}, \lambda)$ fails to have the ISP, whereas the set $\mathcal{A}(\mathcal{K}, t)$ has this property for any $t>0$.

Example 4.1. Let $n=m=2$ and $u_{0}=(2,0) \in \mathbb{R}^{2}$. Consider the sets

$$
\mathcal{K}=\left\{(0, \xi) \in \mathbb{R}^{2}: \xi \in[-1,1]\right\}, \quad U=u_{0}+\bar{B},
$$

and define the functions $f$ and $L$ as

$$
f(x, u)=u, \quad L(x, u)=|u| .
$$

Then the function $L(x, \cdot)$ is very regular on $U$ (even convex), the dynamics $\bar{f}(x, u)=u /|u|$ is well defined and its set of the admissible velocities is the $\operatorname{arc} \bar{F}(x)=\left\{(\cos \alpha, \sin \alpha): \alpha \in\left[-\frac{\pi}{6}, \frac{\pi}{6}\right]\right\}$ for any $x \in \mathbb{R}^{2}$ (see Fig. 1).

So $\bar{f}$ satisfies assumptions (2.2) and (2.4)(iii), and $\bar{F}(x)$ is closed, but it is not convex nor it has the ISP (assumptions (2.4)(i-ii) are not satisfied). In fact, for any $t \geqslant 0$, the attainable set $\overline{\mathcal{A}}(\mathcal{K}, t)$ fails to have the ISP (and in view of Cor. 3.3, the set $\mathcal{A}_{L}(\mathcal{K}, \lambda)$ fails too, for any $\lambda \geqslant 0$ ). On the other hand, $f$ satisfies all the assumptions of Theorem 2.9, and $\mathcal{A}(\mathcal{K}, t)$ has the ISP for any $t>0$. Indeed, define the continuous function $g: \mathbb{R} \times \mathbb{R} \rightarrow \mathbb{R}$,

$$
g(\xi, t)=\left\{\begin{array}{lr}
\sqrt{t^{2}-(|\xi|-1)^{2}} & \xi \in\left[-1-\frac{t}{2},-1\right] \cup\left[1,1+\frac{t}{2}\right], \\
t & \xi \in[-1,1], \\
\frac{\sqrt{3}}{2} t & \text { elsewhere. }
\end{array}\right.
$$



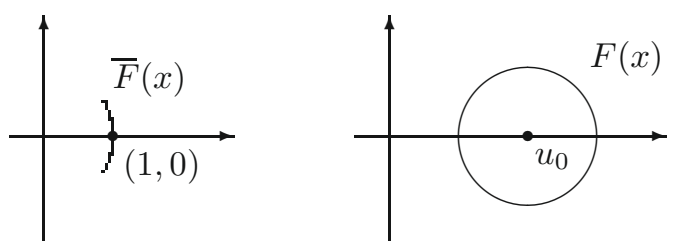

Figure 1. The admissible velocities.
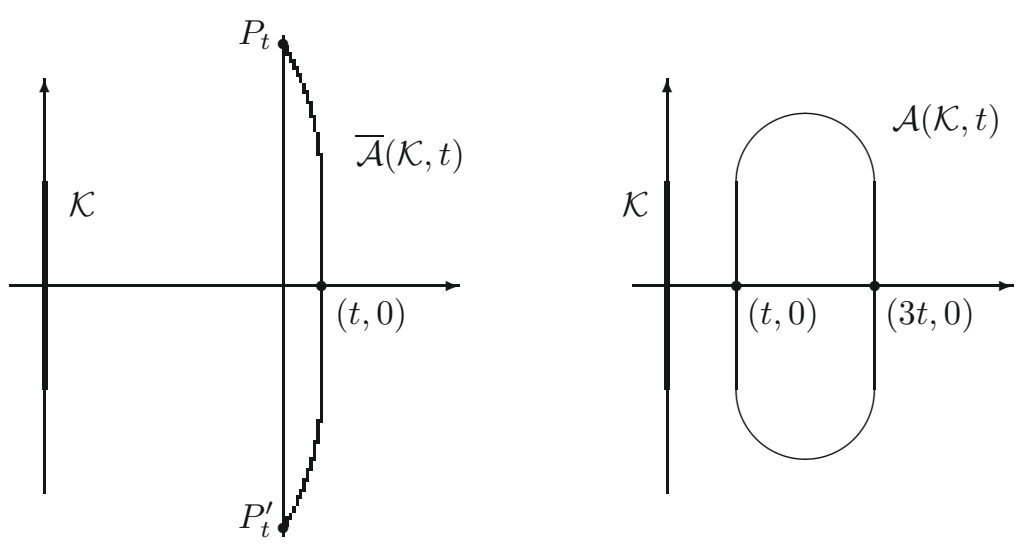

Figure 2. The attainable set.

Then, for any $t \geqslant 0$, the attainable set in time $t$ for $\bar{f}$,

$$
\overline{\mathcal{A}}(\mathcal{K}, t)=\left\{\left(x_{1}, x_{2}\right) \in \mathbb{R}^{2}:-1-\frac{t}{2} \leqslant x_{2} \leqslant 1+\frac{t}{2}, \frac{\sqrt{3}}{2} t \leqslant x_{1} \leqslant g\left(x_{2}, t\right)\right\},
$$

has "bad" corners for the ISP at the points $P_{t}=\left(\frac{\sqrt{3}}{2} t, 1+\frac{t}{2}\right)$ and $P_{t}^{\prime}=\left(\frac{\sqrt{3}}{2} t,-1-\frac{t}{2}\right)$, whereas the attainable set in time $t$ for $f$,

$$
\mathcal{A}(\mathcal{K}, t)=\bigcup_{\xi \in[-1,1]} \bar{B}_{t}((2 t, \xi))
$$

has the ISP of radius $t$ (see Fig. 2).

In order to make $\bar{f}$ satisfying assumptions of Theorem 2.9, on $L$, in addition to assumption (2.3), we will assume that

$$
L \in \mathrm{C}_{l o c}^{1,1}\left(\mathbb{R}^{n}\right)
$$

Remark 4.2. Suppose that $\mathcal{K}$ is compact. For any fixed time $T_{0}>0$ and for any $\lambda_{0}>0$, if Assumptions (2.2), (2.3), (2.4) and (4.1) hold true, then the set $\mathcal{A}(\mathcal{K}, T) \cup \mathcal{A}_{L}(\mathcal{K}, \lambda)$ is contained in a compact set for all $T \in\left[0, T_{0}\right]$ and for all $\lambda \in\left[0, \lambda_{0}\right]$ (in view of Rem. 2.5), and we can assume that there exist constants such that, for any $x, y$ and for all $u \in U$,

$$
\left\{\begin{aligned}
|f(x, u)| & \leqslant M ; \\
|| f_{x}(x, u)-f_{x}(y, u)|| & \leqslant L_{1}|x-y| \\
L(x) & \leqslant \bar{M} ; \\
|L(x)-L(y)| & \leqslant \bar{L}_{2}|x-y| ; \\
\left|L_{x}(x)-L_{x}(y)\right| & \leqslant \bar{L}_{3}|x-y| .
\end{aligned}\right.
$$

So, the dynamics $\bar{f}$ satisfies assumptions (2.2), (2.4) and (2.6). The same simplifying assumptions can be made if our analysis is restricted to a compact set $\mathcal{X}$. 
We have left to find hypothesis guaranteeing that $x \rightsquigarrow \partial \bar{F}(x)$ is a Lipschitz boundary map. But it is very easy, because it is a direct consequence of the same property for the original map $F(x)$.

Proposition 4.3. Let $\mathcal{X} \subset \mathbb{R}^{n}$ be a compact set. Assume that (2.2), (2.3), (2.4) and (4.1) hold true. If $x \rightsquigarrow \partial F(x)$ is a Lipschitz boundary map on $\mathcal{X}$, then $x \rightsquigarrow \partial \bar{F}(x)$ is a Lipschitz boundary map on $\mathcal{X}$.

Remark 4.4. With stronger assumptions, a global Lipschitz regularity can be obtained for $x \rightsquigarrow \partial \bar{F}(x)$ as well as the local result in the above proposition. That is, if the simplifying assumptions (4.2) hold true for any $x, y \in \mathbb{R}^{n}$ and if $x \rightsquigarrow \partial F(x)$ is a Lipschitz boundary map on $\mathbb{R}^{n}$, then $x \rightsquigarrow \partial \bar{F}(x)$ also is a Lipschitz boundary map on $\mathbb{R}^{n}$.

Proof of Proposition 4.3. We want to find $\bar{C}_{0}$ and $\bar{r}_{1}$ such that

$$
\left|\nabla b_{\bar{F}(y)}\left(\bar{v}_{x}\right)-\nabla b_{\bar{F}(x)}\left(\bar{v}_{x}\right)\right| \leqslant \bar{C}_{0}|x-y| \quad \forall \bar{v}_{x} \in \partial \bar{F}(x), \forall y \in B\left(x, \bar{r}_{1}\right) .
$$

Observe that $\nabla b_{\bar{F}(x)}\left(\bar{v}_{x}\right)=\nabla b_{F(x)}\left(v_{x}\right)$, where $v_{x}:=L(x) \bar{v}_{x} \in \partial F(x)$. In fact $\nabla b_{\bar{F}(x)}\left(\bar{v}_{x}\right)$ is the only unit vector $\nu$ such that

$$
\left\langle\nu, \bar{v}-\bar{v}_{x}\right\rangle \leqslant 0 \quad \forall \bar{v} \in \bar{F}(x) .
$$

This implies that $L(x)\left\langle\nu, \bar{v}-\bar{v}_{x}\right\rangle=\left\langle\nu, L(x)\left(\bar{v}-\bar{v}_{x}\right)\right\rangle \leqslant 0$ for all $\bar{v} \in \bar{F}(x)$. Since $F(x)=\{L(x) \bar{v}: \bar{v} \in \bar{F}(x)\}$, we have that $\nu$ is the only unit vector such that $\left\langle\nu, v-v_{x}\right\rangle \leqslant 0$ for all $v \in F(x)$, and then $\nu=\nabla b_{F(x)}\left(v_{x}\right)$.

Furthermore $\nabla b_{\bar{F}(y)}\left(\bar{v}_{x}\right)=\nabla b_{\bar{F}(y)}\left(\pi_{\partial \bar{F}(y)}\left(\bar{v}_{x}\right)\right)=\nabla b_{\bar{F}(y)}\left(\bar{v}_{y}\right)$, where

$$
\bar{v}_{y}:=\pi_{\partial \bar{F}(y)}\left(\bar{v}_{x}\right) \in \partial \bar{F}(y) .
$$

Note that, again, we have $\nabla b_{\bar{F}(y)}\left(\bar{v}_{y}\right)=\nabla b_{F(y)}\left(v_{y}\right)$, where $v_{y}:=L(y) \bar{v}_{y}$. Thus

$$
\left|\nabla b_{\bar{F}(y)}\left(\bar{v}_{x}\right)-\nabla b_{\bar{F}(x)}\left(\bar{v}_{x}\right)\right|=\left|\nabla b_{F(y)}\left(v_{y}\right)-\nabla b_{F(x)}\left(v_{x}\right)\right| ;
$$

where we point out that we don't know if $v_{y}$ is $\pi_{\partial F(y)}\left(v_{x}\right)$ (it is not the case, in general). Due to Proposition 2.7, we have that

$$
\begin{aligned}
\left|\nabla b_{F(y)}\left(v_{y}\right)-\nabla b_{F(x)}\left(v_{x}\right)\right| & \leqslant\left|\nabla b_{F(y)}\left(v_{y}\right)-\nabla b_{F(y)}\left(\pi_{\partial F(y)}\left(v_{x}\right)\right)\right|+\left|\nabla b_{F(y)}\left(\pi_{\partial F(y)}\left(v_{x}\right)\right)-\nabla_{F(x)}\left(v_{x}\right)\right| \\
& =\left|\nabla b_{F(y)}\left(v_{y}\right)-\nabla b_{F(y)}\left(\pi_{\partial F(y)}\left(v_{x}\right)\right)\right|+\left|\nabla b_{F(y)}\left(v_{x}\right)-\nabla b_{F(x)}\left(v_{x}\right)\right| \\
& \leqslant \frac{2}{r_{\mathcal{X}}}\left|v_{y}-\pi_{\partial F(y)}\left(v_{x}\right)\right|+C_{0}|x-y| .
\end{aligned}
$$

Then we have to bound $\left|v_{y}-\pi_{\partial F(y)}\left(v_{x}\right)\right| \leqslant\left|v_{y}-v_{x}\right|+\left|v_{x}-\pi_{\partial F(y)}\left(v_{x}\right)\right|$. But we know that, by Proposition 2.7, $\left|v_{x}-\pi_{\partial F(y)}\left(v_{x}\right)\right|=\left|b_{F(y)}\left(v_{x}\right)\right| \leqslant L_{0}|x-y|$. Moreover

$$
\begin{aligned}
\left|v_{y}-v_{x}\right| & =\left|L(y) \bar{v}_{y}-L(x) \bar{v}_{x}\right| \\
& \leqslant\left|L(y) \bar{v}_{y}-L(y) \bar{v}_{x}\right|+\left|L(y) \bar{v}_{x}-L(x) \bar{v}_{x}\right| \\
& \leqslant L(y)\left|\bar{v}_{y}-\bar{v}_{x}\right|+|\bar{v}(x)| \cdot|L(y)-L(x)| \\
& \leqslant\left(\bar{M} \bar{L}_{0}+\frac{M}{k_{0}} \bar{L}_{2}\right)|x-y|
\end{aligned}
$$

where we used the constants of Remark 4.2, and $\bar{L}_{0}$ is the constant such that $|\bar{f}(x, u)-\bar{f}(y, u)| \leqslant \bar{L}_{0}|x-y|$. For instance we can take $\bar{L}_{0}=\frac{\bar{M} L_{0}+M \bar{L}_{2}}{k_{0}^{2}}$. And this concludes the proof, by taking $\bar{C}_{0}=\frac{2}{r_{\mathcal{X}}}\left(\bar{M} \bar{L}_{0}+\frac{M}{k_{0}} \bar{L}_{2}+L_{0}\right)+$ $C_{0}$, and eventually by reducing $\bar{r}_{1}$ from $r_{1}$ to $\frac{r_{\mathcal{X}}}{2 \bar{L}_{0} \bar{M}}$.

In this way we have already proved one of our main results. 
Theorem 4.5. Let $\mathcal{K} \subseteq \mathbb{R}^{n}$ be a closed set. Assume that (2.2), (2.3), (2.4) and (4.1) hold true, and let $x \rightsquigarrow \partial F(x)$ be a locally Lipschitz boundary map. Then the following are true:

(i) for any $\lambda>0, \mathcal{A}_{L}(\mathcal{K}, \lambda)$ has the ISP at any point of $\partial \mathcal{A}_{L}(\mathcal{K}, \lambda)$ (in general, not uniform);

(ii) for any compact set $\mathcal{X} \subset \mathbb{R}^{n}$, and for any $\lambda_{0}>0$ there exists $\rho=\rho\left(\mathcal{X}, \lambda_{0}\right)$ such that $\mathcal{A}_{L}(\mathcal{K}, \lambda)$ has the ISP of radius $\rho \lambda$ at any point $x \in \partial \mathcal{A}_{L}(\mathcal{K}, \lambda) \cap \mathcal{X}$, for all $\lambda \in\left(0, \lambda_{0}\right)$;

(iii) if $\mathcal{K}$ is a compact set, then for any $\lambda_{0}>0$ there exists $\rho=\rho\left(\lambda_{0}\right)$ such that $\mathcal{A}_{L}(\mathcal{K}, \lambda)$ has the ISP of radius $\rho \lambda$ for all $\lambda \in\left(0, \lambda_{0}\right)$;

(iv) if $L \in \mathrm{C}^{1,1}\left(\mathbb{R}^{n}\right)$, the functions $|f|,|L|$ and $\left|L_{x}\right|$ are globally bounded, (2.4) holds globally, and $x \rightsquigarrow \partial F(x)$ is a Lipschitz boundary map on $\mathbb{R}^{n}$, then for any $\lambda_{0}>0$ there exists $\rho=\rho\left(\lambda_{0}\right)$ such that $\mathcal{A}(\mathcal{K}, \lambda)$ has the ISP of radius $\rho \lambda$ for all $\lambda \in\left(0, \lambda_{0}\right)$.

Proof. Point (iv) follows from Theorem 2.9, Corollary 3.3 and Remark 4.4. Indeed, simplifying assumptions (4.2) are satisfied on $\mathbb{R}^{n}$, and $\bar{f}$ satisfies assumptions of Theorem 2.9.

Point (iii) follows from Remark 4.2, since we can suppose simplifying assumptions (4.2). So, assumptions of point (iv) are satisfied.

To prove point (ii), fix a number $\rho_{0}>0$ and set $\mathcal{X}^{\prime}:=\mathcal{X}+2 \rho_{0} \lambda_{0} \bar{B}$. Note that, for any $\lambda \in\left[0, \lambda_{0}\right]$, if $J(t, x, u)=\lambda$, then $t \in\left[0, \frac{\lambda_{0}}{k_{0}}\right]$. From Remark 2.5 there exists $\hat{R}=\hat{R}\left(\mathcal{X}^{\prime}, \frac{\lambda_{0}}{k_{0}}\right)$ such that, if $y^{x, u}(t) \in \mathcal{A}_{L}(\mathcal{K}, \lambda) \cap \mathcal{X}^{\prime}$ with $J(t, x, u)=\lambda$, then $x \in \mathcal{K} \cap\left(\mathcal{X}^{\prime}+\hat{R} \bar{B}\right)=: \mathcal{K}^{\prime}$. Since $\mathcal{K}^{\prime}$ is compact and $\mathcal{A}_{L}(\mathcal{K}, \lambda) \cap \mathcal{X}^{\prime} \subseteq \mathcal{A}_{L}\left(\mathcal{K}^{\prime}, \lambda\right)$, the conclusion follows from point (iii), possibly reducing $\rho$ to $\rho_{0}$.

Finally, point (i) is a direct consequence of point (ii).

In Theorem 4.5, we need that $x \rightsquigarrow \bar{F}(x)$ is a locally Lipschitz boundary map. In view of Proposition 4.3, it suffices to have the same regularity for $F$. Then we propose a sufficient condition to obtain this Lipschitz regularity for $F$ (and then for $\bar{F}$ ).

Proposition 4.6. Let $\mathcal{X} \subset \mathbb{R}^{n}$ be a compact set. Assume that (2.2), (2.3), (2.4) and (4.1) hold true. Suppose that there exists $C_{2} \geqslant 0$ such that, for all $(x, u) \in \mathcal{X} \times U$ satisfying $f(x, u) \in \partial F(x)+\frac{r}{2} B$,

$$
\left|\left[f_{x}\left(x, u^{\prime}\right)-f_{x}(x, u)\right]^{*} \nabla b_{F(x)}(f(x, u))\right| \leqslant C_{2}\left|f\left(x, u^{\prime}\right)-f(x, u)\right| \quad \forall u^{\prime} \in U .
$$

Then $x \rightsquigarrow \partial \bar{F}(x)$ is a Lipschitz boundary map on $\mathcal{X}$.

For the proof, we refer the reader to Proposition 3.9 of [3].

\section{Application to value function}

We still consider a control system of the form (2.1), i.e.

$$
\left\{\begin{array}{l}
\dot{y}(t)=f(y(t), u(t)), \quad \text { a.e. } t \geqslant 0 \\
y(0)=x
\end{array}\right.
$$

But we look at the system from another point of view. In the previous sections, we were interested to study the trajectories starting from a point $x$ in the set $\mathcal{K}$. In this section we consider the trajectories starting from a generic point $x \in \mathbb{R}^{n}$, and we want to minimize the time (or cost) to arrive at the set $\mathcal{K}$.

Let $\mathcal{K} \subseteq \mathbb{R}^{n}$ a closed set, called target. And let $x \in \mathbb{R}^{n}$ be a point, $u(\cdot)$ a control. We consider the transfer time to $\mathcal{K}$

$$
\tau_{\mathcal{K}}(x, u)=\inf \{t \geqslant 0: y(t ; x, u) \in \mathcal{K}\},
$$

where we set $\tau_{\mathcal{K}}(x, u):=+\infty$ if $y(t ; x, u) \notin \mathcal{K}$ for all $t \geqslant 0$. The minimum time function is $T_{\mathcal{K}}(x)=$ $\inf _{u(\cdot)} \tau_{\mathcal{K}}(x, u)$. 
Let $L: \mathbb{R}^{n} \rightarrow \mathbb{R}$ be the running cost, and consider the cost functional

$$
J(x, u)=\int_{0}^{\tau(x, u)} L(y(t ; x, u)) \mathrm{d} t,
$$

and the value function

$$
V_{\mathcal{K}}(x)=\inf _{u(\cdot)} J(x, u)
$$

We call the level sets of $V_{\mathcal{K}}$, controllable set to $\mathcal{K}$ with cost $\leqslant \lambda$,

$$
\mathcal{C}(\mathcal{K}, \lambda)=\left\{x \in \mathbb{R}^{n}: V_{\mathcal{K}}(x) \leqslant \lambda\right\}
$$

Moreover $\mathcal{C}(\mathcal{K})=\left\{x \in \mathbb{R}^{n}: V_{\mathcal{K}}(x)<+\infty\right\}$ is the controllable set to $\mathcal{K}$.

Remark 5.1. In this set-up, we can take advantage of results of Section 4. If we consider a control system starting from the set $\mathcal{K}$, and with dynamics $\hat{f}=-f$, we have that the associated attainable set with cost $\lambda, \hat{\mathcal{A}}_{L}(\mathcal{K}, \lambda)$, has an interesting relation with the controllable set $\mathcal{C}(\mathcal{K}, \lambda)$. In fact, in general we have that $\hat{\mathcal{A}}_{L}(\mathcal{K}, \lambda) \subseteq \mathcal{C}(\mathcal{K}, \lambda)$, and, in particular,

$$
\partial \mathcal{C}(\mathcal{K}, \lambda) \subseteq \partial \hat{\mathcal{A}}_{L}(\mathcal{K}, \lambda)
$$

Proof. Suppose that $x_{0} \in \partial \mathcal{C}(\mathcal{K}, \lambda)$. In view of continuity of $V_{\mathcal{K}}$, we have that $V_{\mathcal{K}}\left(x_{0}\right)=\lambda$. Then $x_{0} \in \hat{\mathcal{A}}_{L}(\mathcal{K}, \lambda)$. Moreover, $x_{0}$ cannot be in the interior of $\hat{\mathcal{A}}_{L}(\mathcal{K}, \lambda)$. Otherwise there is a ball $B_{\rho}\left(x_{0}\right) \subseteq \hat{\mathcal{A}}_{L}(\mathcal{K}, \lambda)$, i.e. for any $x \in B_{\rho}\left(x_{0}\right)$, we have that $V_{\mathcal{K}}(x) \leqslant \lambda$. Then $B_{\rho}\left(x_{0}\right) \subseteq \mathcal{C}(\mathcal{K}, \lambda)$, and this contradicts $x_{0} \in \partial \mathcal{C}(\mathcal{K}, \lambda)$.

Proposition 5.2. Assume that (2.2), (2.3), (2.4) and (4.1) are satisfied, and that $x \rightsquigarrow \partial F(x)$ is a locally Lipschitz boundary map. Then we have the following:

(i) the level sets of the value function $V_{\mathcal{K}}$ have the Interior Sphere Property at any point (in general, not uniform);

(ii) for any compact set $\mathcal{X} \subset \mathbb{R}^{n}$ and for any $\lambda>0$, there exists $\rho=\rho(\mathcal{X}, \lambda)$ such that the level set of $V_{\mathcal{K}}$, i.e. $\mathcal{C}(\mathcal{K}, \lambda)$, has the Interior Sphere Property of radius $\rho$ for all $x \in \partial \mathcal{C}(\mathcal{K}, \lambda) \cap \mathcal{X}$.

Remark 5.3. Note that, for the level sets of $V_{\mathcal{K}}$, a uniform ISP can be obtained with stronger assumptions, as well as the ISP of the attainable sets in Theorem 4.5.

Proof of Proposition 5.2. Take a point $x \in \partial \mathcal{C}(\mathcal{K}, \lambda)$. In view of Remark 5.1, $x \in \partial \hat{\mathcal{A}}_{L}(\mathcal{K}, \lambda)$. By Theorem 4.5(i) there exist $y_{x} \in \hat{\mathcal{A}}_{L}(\mathcal{K}, \lambda)$ and $\rho>0$ such that

$$
x \in \bar{B}_{\rho}\left(y_{x}\right) \subseteq \hat{\mathcal{A}}_{L}(\mathcal{K}, \lambda)
$$

Again, Remark 5.1 implies that $\bar{B}_{\rho}\left(y_{x}\right) \subseteq \mathcal{C}(\mathcal{K}, \lambda)$, and point (i) is proved. Moreover, from point (ii) of Theorem 4.5 it follows that $\rho$ is constant for $x \in \partial \mathcal{C}(\mathcal{K}, \lambda) \cap \mathcal{X}$.

As in Section 3, we can emphasize the equivalence with a problem depending only on time. We can consider a control system similar to $(2.1)$, but with dynamics $\bar{f}(x, u)=\frac{1}{L(x)} f(x, u)$. Then we have the associated transfer time $\bar{\tau}_{\mathcal{K}}(x, u)$, the functional cost

$$
\bar{J}(x, u)=\int_{0}^{\bar{\tau}_{\mathcal{K}}(x, u)} \mathrm{d} t=\bar{\tau}_{\mathcal{K}}(x, u),
$$

and the value function

$$
\bar{V}_{\mathcal{K}}(x)=\inf _{u(\cdot)} \bar{J}(x, u)=\bar{T}_{\mathcal{K}}(x) .
$$

Finally we have the controllable set to $\mathcal{K}$ in time $\leqslant t, \overline{\mathcal{C}}(\mathcal{K}, t)$, and the controllable set to $\mathcal{K}, \overline{\mathcal{C}}(\mathcal{K})$ 
Proposition 5.4. Assume that (2.2) and (2.3) are satisfied. Then we have the following:

(i) $J(x, u)=\bar{\tau}_{\mathcal{K}}\left(x, \varphi_{2}(u)\right)$;

(ii) $V_{\mathcal{K}}(x)=\bar{T}_{\mathcal{K}}(x)$;

(iii) $\mathcal{C}(\mathcal{K}, \lambda)=\overline{\mathcal{C}}(\mathcal{K}, \lambda)$ and $\mathcal{C}(\mathcal{K})=\overline{\mathcal{C}}(\mathcal{K})$

where $\varphi$ is the function defined in (3.4).

Proof. Set $\sigma(x, u)=\int_{0}^{\tau_{\mathcal{K}}(x, u)} L\left(y^{x, u}(t)\right) \mathrm{d} t$. In view of (3.5) we have that the trajectory $\varphi_{1}\left(y^{x, u}\right)$ is defined on $[0, \sigma(x, u)]$, and

$$
J(x, u)=\int_{0}^{\tau_{\mathcal{K}}(x, u)} L\left(y^{x, u}(t)\right) \mathrm{d} t=\sigma(x, u) .
$$

On the other hand, by construction,

$$
y^{x, u}\left(\left[0, \tau_{\mathcal{K}}(x, u)\right)\right) \cap \mathcal{K}=\emptyset \quad \Longrightarrow \quad \varphi_{1}\left(y^{x, u}\right)([0, \sigma(x, u))) \cap \mathcal{K}=\emptyset,
$$

and $\varphi_{1}\left(y^{x, u}\right)(\sigma(x, u))=y^{x, u}\left(\tau_{\mathcal{K}}(x, u)\right) \in \mathcal{K}$. Then

$$
J(x, u)=\sigma(x, u)=\bar{\tau}_{\mathcal{K}}\left(x, \varphi_{2}(u)\right) .
$$

Hence (i) is proved, and (ii) and (iii) follow obviously.

We can apply these equivalence results to obtain regularity properties for the value function $V_{\mathcal{K}}$. For the minimum time function $T_{\mathcal{K}}$ it is possible to obtain local semiconcavity in $\mathcal{C}(\mathcal{K}) \backslash \mathcal{K}$, provided that control system (2.1) satisfies the Petrov condition

$$
\exists \mu>0 \text { such that } \min _{u \in U}\langle f(x, u), p\rangle<-\mu|p|, \quad \forall x \in \partial \mathcal{K}, \forall p \in N_{\mathcal{K}}(x) .
$$

In fact, jointly with the ISP of target $\mathcal{K}$, condition (5.1) yields that $T_{\mathcal{K}} \in \mathrm{SC}_{\text {loc }}(\mathcal{C}(\mathcal{K}) \backslash \mathcal{K})$ (see [4]). Moreover, using Remark 5.1 and Proposition 5.2, it is possible to obtain semiconcavity of $T_{\mathcal{K}}$ also in the case of more general target $\mathcal{K}$. An easy adaptation of Theorem 5.2 of [3] yields the following proposition.

Proposition 5.5. Suppose that assumptions (2.2), (2.4) and (5.1) are satisfied. If $x \rightsquigarrow \partial F(x)$ is a locally Lipschitz boundary map, then $T_{\mathcal{K}}$ is locally semiconcave in $\mathcal{C}(\mathcal{K}) \backslash \mathcal{K}$.

As an immediate consequence, due to Proposition 5.4, we have that $V_{\mathcal{K}}$ is locally semiconcave in $\mathcal{C}(\mathcal{K}) \backslash \mathcal{K}$.

Theorem 5.6. Suppose that assumptions (2.2), (2.3), (2.4), (4.1) and (5.1) are satisfied. If $x \rightsquigarrow \partial F(x)$ is a locally Lipschitz boundary map, then the value function $V_{\mathcal{K}}$ is locally semiconcave in $\mathcal{C}(\mathcal{K}) \backslash \mathcal{K}$.

Remark 5.7. We point out that Sinestrari [8], in a similar set-up, proves local semiconcavity for $V_{\mathcal{K}}$. He proves local semiconcavity with a direct approach and, as a consequence, the ISP of the level set is obtained (so, requesting controllability assumption (5.1)). On the contrary, this paper is focused on the Interior Sphere Property of the attainable sets and of the level sets of $V_{\mathcal{K}}$ (without assuming (5.1)), and the equivalence between the minimum time $\bar{T}_{\mathcal{K}}$ and the value function $V_{\mathcal{K}}$.

However we want to emphasize some differences on the assumptions required to obtain semiconcavity for $V_{\mathcal{K}}$. In particular, he requires a $\mathrm{C}^{1}$ regularity and a local semiconcavity for the running cost $L$, and a nondegeneracy property for $f$, i.e.

$$
\left\|f_{x}\left(x, u^{\prime}\right)-f_{x}(x, u)\right\| \leqslant C\left|f\left(x, u^{\prime}\right)-f(x, u)\right| .
$$

Note that, on the one hand, this nondegeneracy property is stronger than boundary Lipschitz continuity that we require on the map $x \rightsquigarrow \partial F(x)$ (even a little bit more than sufficient condition in Prop. 4.6). On the other hand our assumption (4.1) on $L$ is stronger than those in [8]. Indeed $\mathrm{C}_{\text {loc }}^{1,1}$ regularity implies both local semiconcavity and local semiconvexity. 


\section{Perimeter estimates}

As an easy consequence of the previous sections and of the results in [2], we have perimeter estimates in the case of some special control systems.

We restrict our attention to a control system of the form

$$
\left\{\begin{array}{l}
\dot{y}(t)=f(y(t)) u(t), \quad u(t) \in \bar{B} \\
y(0)=x
\end{array}\right.
$$

with $f: \mathbb{R}^{n} \rightarrow \mathbb{R}^{n \times n}$, and $\mathcal{K} \subset \mathbb{R}^{n}$ a nonempty compact set. In this section, with some extra assumption on $f$ and $L$, we show that the attainable set $\mathcal{A}_{L}(\mathcal{K}, \lambda)$ is a set of finite perimeter (in the sense of De Giorgi), and that the growth of the perimeter is controlled by the cost $\lambda$.

For the reader's convenience we briefly introduce the basic instruments, but for details we refer the reader to [7].

Let $\mathcal{I} \subseteq \mathbb{R}^{n}$ be an open set.

Definition 6.1. A function $g \in \mathrm{L}^{1}(\mathcal{I})$ has bounded variation in $\mathcal{I}$ if

$$
\sup \left\{\int_{\mathcal{I}} g \operatorname{div} \varphi \mathrm{d} x: \varphi \in \mathrm{C}_{c}^{1}\left(\mathcal{I}, \mathbb{R}^{n}\right),|\varphi| \leqslant 1\right\}<\infty .
$$

Definition 6.2. A measurable set $E \subseteq \mathbb{R}^{n}$ has finite perimeter in $\mathcal{I}$ if the characteristic function $\chi_{E}$ has bounded variation in $\mathcal{I}$.

The total variation of $g$ in $\mathcal{I}$ is

$$
\|D g\|(\mathcal{I})=\sup \left\{\int_{\mathcal{I}} g \operatorname{div} \varphi \mathrm{d} x: \varphi \in \mathrm{C}_{c}^{1}\left(\mathcal{I}, \mathbb{R}^{n}\right),|\varphi| \leqslant 1\right\}
$$

and the perimeter of a measurable set $E$ in $\mathcal{I}$ is given by $P(E, \mathcal{I}):=\left\|D \chi_{E}\right\|(\mathcal{I})$. Clearly, these definitions can be easily extended to the Borel sets.

For sets of finite perimeter $E$, one can introduce the essential boundary $\partial^{*} E$, in which, for all point $x$, the unit normal $\nu_{E}(x)$ coincides with the local integral average of the normals on the boundary. The measure theoretic boundary $\partial_{*} E$ is the set of points $x \in \mathbb{R}^{n}$ such that

$$
\limsup _{r \rightarrow 0^{+}} \frac{|B(x, r) \cap E|}{r^{n}}>0 \text { and } \quad \limsup _{r \rightarrow 0^{+}} \frac{|B(x, r) \backslash E|}{r^{n}}>0 .
$$

And it is a known fact that

$$
\partial^{*} E \subseteq \partial_{*} E, \quad \mathcal{H}^{n-1}\left(\partial_{*} E \backslash \partial^{*} E\right)=0 .
$$

Finally, the perimeter of a set $E$ in an open set $\mathcal{I}$ coincides with the restriction of $\mathcal{H}^{n-1}$ to $\partial^{*} E$, calculated on $\mathcal{I}$. For instance, if $E$ is a smooth, open subset of $\mathbb{R}^{n}$,

$$
P(E, \mathcal{I})=\mathcal{H}^{n-1}(\partial E \cap \mathcal{I}) .
$$

Since the attainable set in time $t$ has the ISP, then $\mathcal{A}(\mathcal{K}, t)$ is a set of finite perimeter (see [1]). Moreover we have the following perimeter estimates (see [2]).

Proposition 6.3. Let be $f \in \mathrm{C}^{2}\left(\mathbb{R}^{n} ; \mathbb{R}^{n \times n}\right)$, the matrix $f(x)$ invertible for any $x \in \mathbb{R}^{n}$, and let $f$ and $f^{-1}$ be globally bounded. Then, for any $T>0 \mathcal{A}(\mathcal{K}, T)$ has finite perimeter. Moreover there are constants $c_{1}$ and $c_{2}$ such that, for any $t_{1}, t_{2}$ with $0<t_{1}<t_{2} \leqslant T$, we have

$$
\mathcal{H}^{n-1}\left(\partial^{*} \mathcal{A}\left(\mathcal{K}, t_{2}\right)\right) \leqslant C\left(\frac{t_{2}}{t_{1}}\right)^{c_{2}} \mathrm{e}^{c_{1}\left(t_{2}-t_{1}\right)} \mathcal{H}^{n-1}\left(\partial^{*} \mathcal{A}\left(\mathcal{K}, t_{1}\right)\right) .
$$


In the same way of the Section 4, we can take advantage of this result for the sets $\mathcal{A}_{L}(\mathcal{K}, \lambda)$. At this aim we have to find the assumptions on $f(\cdot)$ and $L(\cdot)$ that provides the assumptions of this proposition. Then we need that:

$$
\left\{\begin{aligned}
\text { (i) } & f, L \text { are of class } \mathrm{C}^{2} \\
\text { (ii) } & f \text { is invertible } \\
\text { (iii) } & f, f^{-1}, L \text { are bounded, and } L(x) \geqslant k>0 \text { for all } x \in \mathbb{R}^{n} .
\end{aligned}\right.
$$

Hence, in view of Corollary 3.3 and Proposition 6.3, we have perimeter estimates also for the attainable sets with cost $\lambda$.

Proposition 6.4. Assume (6.2). Then, for any $\Lambda>0$, there are constants $c_{1}, c_{2}$ such that for any $0<\lambda_{1}<$ $\lambda_{2} \leqslant \Lambda$

$$
\mathcal{H}^{n-1}\left(\partial^{*} \mathcal{A}_{L}\left(\mathcal{K}, \lambda_{2}\right)\right) \leqslant C\left(\frac{\lambda_{2}}{\lambda_{1}}\right)^{c_{2}} \mathrm{e}^{c_{1}\left(\lambda_{2}-\lambda_{1}\right)} \mathcal{H}^{n-1}\left(\partial^{*} \mathcal{A}_{L}\left(\mathcal{K}, \lambda_{1}\right)\right) .
$$

Acknowledgements. I thank Piermarco Cannarsa for having introduced me in the subject, for the fruitful discussions and for the numerous suggestions during the preparation of the paper. I thank the anonymous referees too, for their helpful comments and suggestions.

\section{REFERENCES}

[1] O. Alvarez, P. Cardaliaguet and R. Monneau, Existence and uniqueness for dislocation dynamics with nonnegative velocity. Interfaces Free Bound. 7 (2005) 415-434.

[2] P. Cannarsa and P. Cardaliaguet, Perimeter estimates for the reachable set of control problems. J. Convex Anal. 13 (2006) 253-267.

[3] P. Cannarsa and H. Frankowska, Interior sphere property of attainable sets and time optimal control problems. ESAIM: COCV 12 (2006) 350-370.

[4] P. Cannarsa and C. Sinestrari, Convexity properties of the minimun time function. Calc. Var. Partial Differential Equations 3 (1995) 273-298.

[5] P. Cannarsa and C. Sinestrari, Semiconcave Functions, Hamilton-Jacobi Equations and Optimal Control. Birkhauser, Boston (2004).

[6] P. Cannarsa, C. Pignotti and C. Sinestrari, Semiconcavity for optimal control problems with exit time. Discrete Contin. Dynam. Systems 6 (2000) 975-997.

[7] L.C. Evans and F. Gariepy, Measure Theory and Fine Properties of Functions, Studies in Advanced Mathematics. Boca Raton (1992).

[8] C. Sinestrari, Semiconcavity of the value function for exit time problems with nonsmooth target. Commun. Pure Appl. Anal. 3 (2004) 757-774. 